\title{
UK Renal Registry 19th Annual Report: Chapter 10 Epidemiology of Reported Infections in Patients Receiving Dialysis in England between January 2015 and December 2015: a Joint Report from Public Health England and the UK Renal Registry
}

\author{
Lisa Crowley ${ }^{\mathrm{a}}$, Stephanie MacNeill ${ }^{\mathrm{bc}}$, Shona Methven ${ }^{\mathrm{d}}$, Olisaeloka Nsonwu ${ }^{\mathrm{e}}$, \\ John Davies ${ }^{\mathrm{e}}$, Fergus J Caskey ${ }^{\text {bcf }}$, Richard Fluck ${ }^{\mathrm{g}}$, Catherine Byrne ${ }^{\mathrm{h}}$ \\ ${ }^{a}$ Royal Shrewsbury Hospital, Shrewsbury, UK; ${ }^{\text {DUK Renal Registry, Bristol, UK; ' } \mathrm{C} c h o o l}$ of Social and Community Medicine, \\ University of Bristol, Bristol, UK; ${ }^{d}$ Aberdeen Royal Infirmary, Aberdeen, UK; ${ }^{e}$ Public Health England, London, UK; \\ ${ }^{f}$ North Bristol NHS Trust, Bristol, UK; ${ }^{9}$ Royal Derby Hospital, Derby, UK; ${ }^{\text {h}}$ Nottingham University Hospitals NHS Trust, \\ Nottingham, UK
}

\section{Keywords}

Clostridium difficile · Dialysis · Epidemiology · Escherichia coli . Established renal failure - Infection · MRSA . MSSA . Staphylococcus

\section{Summary}

- Between January 2015 and December 2015 there were a total of 31 episodes of Methicillin Resistant Staphylococcus aureus (MRSA) bacteraemia in patients receiving dialysis for end stage renal disease.

- The rate of MRSA episodes per 100 dialysis patient years was 0.13 compared to 0.15 the previous year.
- Rates of Methicillin Sensitive Staphyloccoccus aureus (MSSA) continued their gradual increase with a rate of 2.35 per 100 patient years compared with 2.26 the year before. This was a result of 560 episodes of bloodstream infection between January and December.

- Rates of Clostridium difficile infection (CDI) were stable with 245 recorded episodes giving a rate of 1.03 per 100 patient years.

- Escherichia coli (E.coli) infections occurred at a rate of 1.7 per 100 dialysis patient years, an increase on the previous year's rate of 1.49 .

- As found in previous years, a tunnelled catheter was associated with a higher number of infection episodes than other forms of access in those patients with a staphylococcal bacteraemia.

\section{KARGER}

Fax +4161306 1234 E-Mail karger@karger.com www.karger.com/nef
This article is licensed under the Creative Commons AttributionNonCommercial-NoDerivatives 4.0 International License (CC BYNC-ND) (http://www.karger.com/Services/OpenAccessLicense), Usage and distribution for commercial purposes as well as any distribution of modified material requires written permission.
Lisa Crowley

UK Renal Registry, Southmead Hospital, Southmead Road,

Bristol, BS10 5NB, UK

Email: renalregistry@renalregistry.nhs.uk 


\section{Introduction}

Infection remains one of the leading causes of death in patients receiving renal replacement therapy (RRT) for established renal failure (ERF) [1]. The causes of these high rates of systemic infection are multi-factorial and include an impaired immune system and the type of vascular access used [2]. This chapter covers reporting for Methicillin Resistant Staphylococcus aureus (MRSA), Methicillin Sensitive Staphylococcus aureus (MSSA) and Escherichia coli (E Coli) bloodstream infections as well as episodes of Clostridium difficile infection (CDI) in patients receiving dialysis for ERF. These infections are subject to mandatory reporting to Public Health England (PHE) and previous UK Renal Registry reports have detailed the epidemiology of these infections in dialysis patients.

\section{Methods}

The reporting of MRSA, MSSA, E Coli and CDI episodes to PHE is mandatory, however the completion of data relating to whether patients are in established renal failure is voluntary and depends on the data entry policy of each individual trust. The methods used for reporting of infections to PHE have been detailed in previous registry reports [3]. To account for potential differences in reporting policy, the 2015 UKRR report introduced a new method to standardise the case identification process by linking the UKRR database of patients receiving dialysis to the PHE database of reported positive blood cultures. This process is outlined in more detail in the report from that year [4]. Linked data were again validated by securely emailing clinical or infection control leads at each renal centre and asking them to confirm the following:

1 That each of the cases in the PHE file was correct, i.e. that it related to a dialysis patient receiving treatment at their centre at the time of the infection and

a Removing any cases that occurred in patients not on dialysis and receiving treatment at their centre at the time of the infection

b Adding any cases that were not known to PHE but occurred in patients on dialysis and receiving treatment at their centre at the time of the infection

2 The dialysis modality

3 For MRSA and MSSA bloodstream infections to provide details on the access in use at the time of the infection.

PHE reports individual blood culture results. However this report details individual infection episodes; repeated positive blood cultures within a four-week timeframe are treated as a single infection episode, beyond four weeks they are treated as a new episode or re-infection. Centre specific rates for each infection are presented per 100 dialysis patient years. The denominator for this rate was calculated for each centre by summing the number of days that each dialysis patient contributed between the 1st of January and 31st of December 2015. When calculating the modality specific rates, the number of days that every dialysis patient spent on each modality during the collection period was totalled. The number of patient years at risk by access type was estimated using data from the 2015 dialysis access audit. The percentage of prevalent patients on each form of vascular access on 31st December 2015 was multiplied by the total number of patients on Haemodialyis (HD) on 31st December 2015 to give an estimate of the overall number of patient years at risk.

Finally, in order to adjust for variation in precision of the estimated rate, the rate of bacteraemia/CDI per 100 dialysis patient years has been plotted against the centre size in a funnel plot. This has been plotted for each infection.

The last UKRR report covered the period between May 2013 and April 2014. This year data are presented between January 2015 and December 2015 in order to bring the data collection period in this chapter in line with the rest of the UKRR report. This year's report is the first opportunity to directly compare data collected using the new linkage method across years.

\section{Results}

Table 10.1 displays the number of positive blood cultures reported to PHE and the final number following the validation process. Centres added 25 infection episodes this year in comparison with 17 last year. Thirteen episodes were not confirmed by centres as being associated with a dialysis patient during the validation process.

Table 10.2 shows the overall number of episodes for each infection in the period covered by the report. It also shows the split between dialysis modalities and the overall rate per 100 dialysis patient years.

Centre level data are shown in table 10.3.

Table 10.1. Number of infectious episodes reported to Public Health England (PHE) and validated by renal centres in 2015

\begin{tabular}{lcccc}
\hline & MRSA & MSSA & CDI & E.coli \\
\hline $\begin{array}{l}\text { Number of infectious } \\
\text { episodes reported to PHE }\end{array}$ & 29 & 580 & 281 & 426 \\
$\begin{array}{l}\text { Number of episodes rejected } \\
\text { by centres during validation }\end{array}$ & 0 & 1 & 2 & 10 \\
$\begin{array}{l}\text { Number of episodes added } \\
\text { by centres during validation }\end{array}$ & 2 & 13 & 2 & 10 \\
$\begin{array}{l}\text { Number of duplicate } \\
\text { episodes reported to PHE }\end{array}$ & 0 & 32 & 36 & 21 \\
$\begin{array}{l}\text { Total number of episodes } \\
\text { after validation process }\end{array}$ & $\mathbf{3 1}$ & $\mathbf{5 6 0}$ & $\mathbf{2 4 5}$ & $\mathbf{4 0 5}$ \\
\hline
\end{tabular}


Table 10.2. Overall number of episodes by modality and rate per 100 dialysis patient years in 2015

\begin{tabular}{|c|c|c|c|c|}
\hline & \multicolumn{4}{|c|}{ Infection } \\
\hline & MRSA & MSSA & CDI & E.coli \\
\hline Total & 31 & 560 & 245 & 405 \\
\hline $\mathrm{HD}$ & 31 & 548 & 216 & 376 \\
\hline $\mathrm{PD}$ & & 12 & 29 & 29 \\
\hline Total & $0.13(0.09-0.18)$ & $2.35(2.16-2.56)$ & $1.03(0.9-1.17)$ & $1.7(1.54-1.88)$ \\
\hline HD & $0.15(0.1-0.21)$ & $2.65(2.43-2.88)$ & $1.04(0.91-1.19)$ & $1.82(1.64-2.01)$ \\
\hline $\mathrm{PD}$ & 0 & $0.39(0.2-0.67)$ & $0.93(0.62-1.34)$ & $0.93(0.62-1.34)$ \\
\hline
\end{tabular}

HD haemodialyis

PD peritoneal dialysis

Methicillin resistant Staphylococcus aureus

There were a total of 31 MRSA infection episodes in the period covered by the report at a rate of $0.13(95 \%$ CI 0.09-0.18) per 100 dialysis patient years (table 10.2). This is very similar to last year's rate of 0.15 per 100 patient years, and as shown by the box and whisker plot of MRSA rates over the last few years (figure 10.1), continues to suggest that the MRSA rate amongst dialysis patients has reached a plateau. All recorded MRSA episodes occurred in patients receiving haemodialysis.

Only one centre (Bradford) had an MRSA rate in excess of 1.0 per 100 dialysis patient years which is the Renal Association audit standard. The funnel plot in figure 10.2 plots each centre's estimated rate against the number of patient years to take into account the greater variation expected as centre size decreases, however the low numbers of episodes at each centre makes the comparison of rates unreliable.

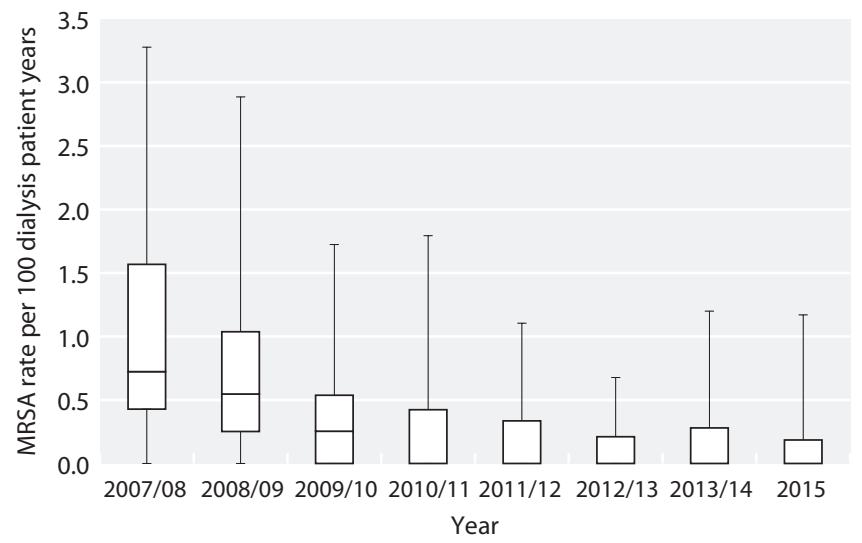

Fig. 10.1. Box and whisker plot of MRSA rates by renal centre per 100 dialysis patient years, by reporting year

Epidemiology of infection in dialysis patients
Methicillin sensitive Staphylococcus aureus

In total, there were 560 episodes of MSSA infection in the period covered at a rate of 2.35 (95\% CI 2.16-2.56) per 100 dialysis patient years. This represents a slight increase from last year's rate of 2.23 per 100 dialysis patient years. Figure 10.3 demonstrates the trend in MSSA infection rates. In previous years the methods of data collection and validation have varied making absolute comparisons difficult, however this collection and validation method was identical to that used last year allowing direct comparisons to be made.

There was considerable variation between centres in the rate of MSSA bloodstream infection per 100 dialysis patient years with a low figure of 0.30 and a high figure of 7.65. The funnel plot (figure 10.4) allows comparison between centres' estimated rates. There were also differences by dialysis modality where the rate of infection is

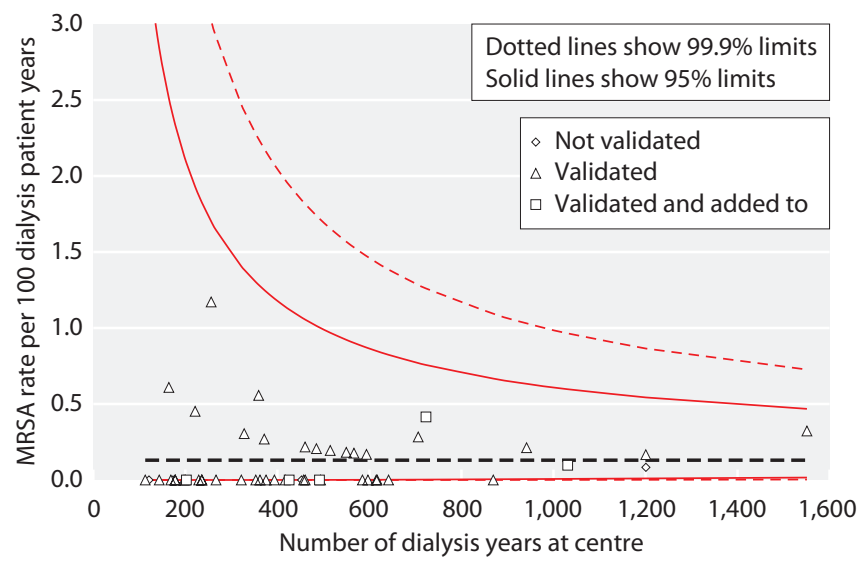

Fig. 10.2. Funnel plot of the MRSA bacteraemia rate per 100 dialysis patient years by renal centre, 1st January 2015 to 31st December 2015

Dotted line depicts rate for whole cohort

Nephron 2017;137(suppl1):251-258 
Table 10.3. Number and rate of infectious episodes in patients with established renal failure by renal centre

\begin{tabular}{|c|c|c|c|c|c|c|c|c|c|}
\hline \multirow[b]{2}{*}{ Centre } & \multirow{2}{*}{$\begin{array}{c}\text { Dialysis } \\
\text { patient } \\
\text { years }\end{array}$} & \multicolumn{4}{|c|}{ Number of episodes $(1 / 01 / 2015-31 / 12 / 2015)$} & \multicolumn{4}{|c|}{ Rate per 100 dialysis patient years } \\
\hline & & MRSA & MSSA & CDI & E.coli & MRSA & MSSA & CDI & E.coli \\
\hline B QEH & 1,201 & 2 & 23 & 12 & 17 & 0.17 & 1.92 & 1.00 & 1.42 \\
\hline Basldn & 197 & 0 & 1 & 1 & 0 & 0.00 & 0.51 & 0.51 & 0.00 \\
\hline Bradfd & 256 & 3 & 10 & 1 & 8 & 1.17 & 3.90 & 0.39 & 3.12 \\
\hline Camb* & & & & & & & & & \\
\hline Carlis & 113 & 0 & 6 & 1 & 2 & 0.00 & 5.32 & 0.89 & 1.77 \\
\hline Carsh & 942 & 2 & 13 & 2 & 14 & 0.21 & 1.38 & 0.21 & 1.49 \\
\hline Chelms & 164 & 1 & 1 & 1 & 3 & 0.61 & 0.61 & 0.61 & 1.83 \\
\hline Colchr & 121 & 0 & 4 & 0 & 3 & 0.00 & 3.31 & 0.00 & 2.48 \\
\hline Covnt & 457 & 0 & 8 & 6 & 5 & 0.00 & 1.75 & 1.31 & 1.09 \\
\hline Exeter & 515 & 1 & 8 & 10 & 15 & 0.19 & 1.55 & 1.94 & 2.91 \\
\hline Glouc & 267 & 0 & 8 & 4 & 3 & 0.00 & 3.00 & 1.50 & 1.12 \\
\hline Hull & 426 & 0 & 16 & 2 & 10 & 0.00 & 3.76 & 0.47 & 2.35 \\
\hline Ipswi & 173 & 0 & 7 & 3 & 2 & 0.00 & 4.04 & 1.73 & 1.16 \\
\hline Kent & 485 & 1 & 16 & 2 & 8 & 0.21 & 3.30 & 0.41 & 1.65 \\
\hline L Barts & 1,201 & 1 & 37 & 10 & 24 & 0.08 & 3.08 & 0.83 & 2.00 \\
\hline L Guys & 706 & 2 & 18 & 8 & 14 & 0.28 & 2.55 & 1.13 & 1.98 \\
\hline L Kings & 642 & 0 & 3 & 0 & 1 & 0.00 & 0.47 & 0.00 & 0.16 \\
\hline L Rfree & 869 & 0 & 9 & 10 & 15 & 0.00 & 1.04 & 1.15 & 1.73 \\
\hline L St.G & 372 & 1 & 7 & 4 & 3 & 0.27 & 1.88 & 1.08 & 0.81 \\
\hline L West & 1,551 & 5 & 31 & 15 & 28 & 0.32 & 2.00 & 0.97 & 1.81 \\
\hline Nottm & 461 & 0 & 11 & 5 & 17 & 0.00 & 2.39 & 1.09 & 3.69 \\
\hline Oxford & 550 & 1 & 14 & 9 & 8 & 0.18 & 2.55 & 1.64 & 1.46 \\
\hline Plymth & 169 & 0 & 2 & 3 & 5 & 0.00 & 1.19 & 1.78 & 2.97 \\
\hline Ports & 723 & 3 & 23 & 5 & 17 & 0.41 & 3.18 & 0.69 & 2.35 \\
\hline Prestn & 617 & 0 & 12 & 7 & 10 & 0.00 & 1.95 & 1.13 & 1.62 \\
\hline Redng & 375 & 0 & 10 & 3 & 9 & 0.00 & 2.66 & 0.80 & 2.40 \\
\hline Salford & 494 & 0 & 15 & 7 & 4 & 0.00 & 3.04 & 1.42 & 0.81 \\
\hline Sheff & 615 & 0 & 10 & 7 & 8 & 0.00 & 1.63 & 1.14 & 1.30 \\
\hline Shrew & 236 & 0 & 6 & 3 & 6 & 0.00 & 2.55 & 1.27 & 2.55 \\
\hline Stevng & 594 & 1 & 12 & 3 & 3 & 0.17 & 2.02 & 0.51 & 0.51 \\
\hline Sthend & 143 & 0 & 8 & 0 & 3 & 0.00 & 5.58 & 0.00 & 2.09 \\
\hline Stoke & 417 & 0 & 6 & 9 & 8 & 0.00 & 1.44 & 2.16 & 1.92 \\
\hline Sund & 236 & 0 & 7 & 3 & 0 & 0.00 & 2.96 & 1.27 & 0.00 \\
\hline Truro & 178 & 0 & 6 & 5 & 2 & 0.00 & 3.37 & 2.81 & 1.12 \\
\hline Wirral & 221 & 1 & 8 & 4 & 2 & 0.45 & 3.62 & 1.81 & 0.90 \\
\hline Wolve & 394 & 0 & 7 & 3 & 5 & 0.00 & 1.78 & 0.76 & 1.27 \\
\hline York & 178 & 0 & 7 & 1 & 4 & 0.00 & 3.94 & 0.56 & 2.25 \\
\hline England & 23,794 & 31 & 560 & 245 & 405 & 0.13 & 2.35 & 1.03 & 1.70 \\
\hline
\end{tabular}

${ }^{*}$ Cambridge were unable to submit data to the UKRR for 2015 


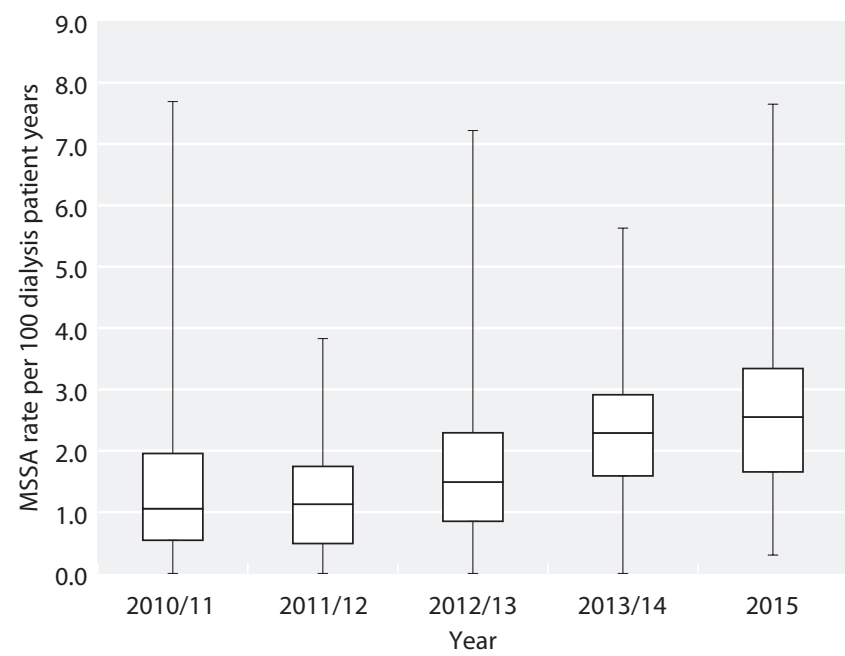

Fig. 10.3. Box and whisker plot of MSSA rates by renal centre per 100 dialysis patient years, by reporting year

seven-fold higher in the HD population than in the PD population (table 10.2).

\section{Vascular access type}

For MRSA bacteraemia episodes, access data were available for 20 of the 31 infection episodes. Of these 20 episodes, 14 occurred while patients were using a central venous catheter as access, five occurred in patients with an arteriovenous fistula and one occurred in a patient with an arteriovenous graft. Of the 560 episodes with a confirmed MSSA, vascular access data were provided for 453 episodes. Two hundred and seventy one episodes occurred while patients had either a tunnelled or nontunnelled central venous catheter (almost 60\% of the

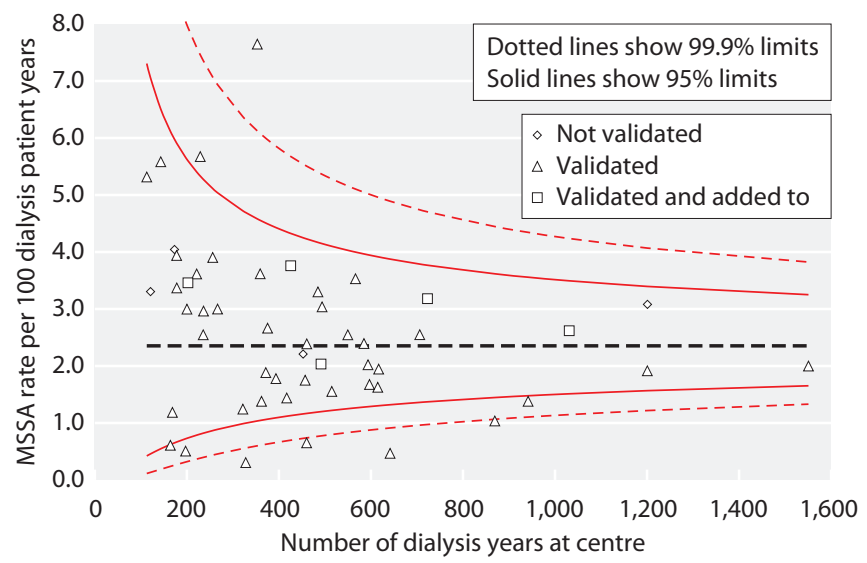

Fig. 10.4. Funnel plot of the MSSA bacteraemia rate per 100 dialysis patient years by renal centre, 1st January 2015 to 31st December 2015

Dotted line depicts rate for whole cohort

Epidemiology of infection in dialysis patients
Table 10.4. Type of dialysis access in use at the time of infection for HD patients

\begin{tabular}{lrrrrr}
\hline & \multicolumn{5}{c}{$\begin{array}{c}\text { Number of episodes } \\
(1 / 01 / 2015-31 / 12 / 2015)\end{array}$} \\
\cline { 2 - 6 } & AVF & AVG & CVC & PD & No data \\
\hline $\begin{array}{l}\text { Estimated number of } \\
\text { patient years at risk }\end{array}$ & 12,938 & 1,368 & $7,026^{*}$ & 3,093 \\
MRSA & 7 & 1 & 17 & & 6 \\
MSSA & 153 & 20 & 271 & 9 & 107 \\
\hline
\end{tabular}

* Only data for combined non-tunnelled and tunnelled catheters available

AVF arteriovenous fistula; AVG arteriovenous graft; CVC central venous catheter; PD peritoneal dialysis

total episodes). The access data for these infections are summarised in table 10.4. The estimated number of patient years at risk is also shown. Absolute risk rates cannot be calculated because vascular access has, until now, only been captured at one time point every 12 months, so the time at risk while exposed to each form of access was not available. Instead the estimated number of patient years at risk is given based on the distribution of access types using data from the 33 centres in England who provided prevalent access data in the 2015 dialysis access audit return. This distribution was then applied to the total number of patients on HD in England on 31st December 2015 to give an overall estimate for England.

\section{Clostridium difficile}

There were a total of 245 Clostridium difficile infection episodes reported this year representing a rate of 1.03 per 100 dialysis patient years. This is comparable to results in last year's report where there were 247 infection episodes representing a rate of 1.05 per 100 dialysis patient years. Rates were comparable between the PD and HD populations (table 10.2). Three centres reported no episodes, the highest reported rate was 2.81 per 100 dialysis patient years. The funnel plot in figure 10.5 demonstrates that no centres were exceeding their estimate rate.

\section{Escherichia coli}

A total of 405 episodes of E.coli bacteraemia were reported in the time period covered by this report, giving a national rate of 1.7 per 100 patient years (95\% CI $1.54-$ 1.88). This compares with a rate of 1.49 reported last year. There was considerable variation once again between centres, two of which did not report any episodes. The highest rate was 3.69 per 100 patient years. Figure 10.6

Nephron 2017;137(suppl1):251-258 


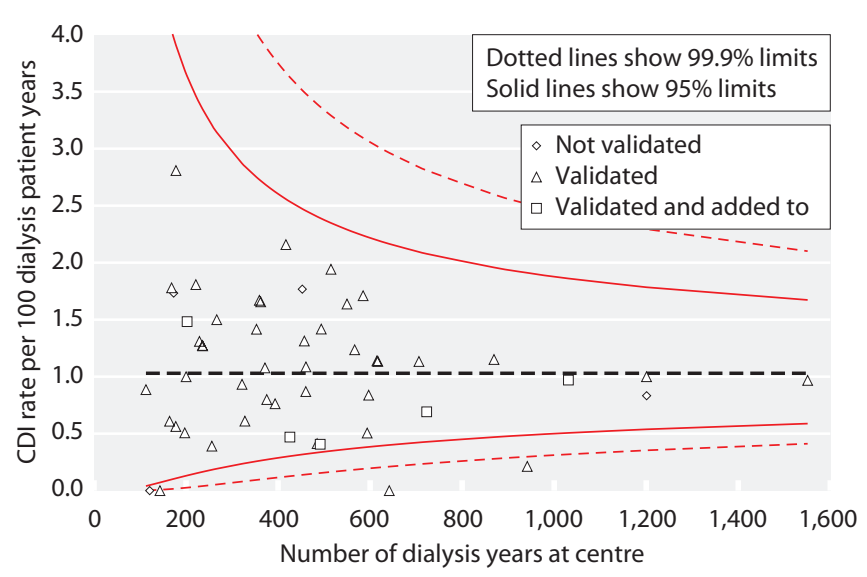

Fig. 10.5. Funnel plot of the CDI rate per 100 dialysis patient years by renal centre, 1st January 2015 to 31st December 2015 Dotted line depicts rate for whole cohort

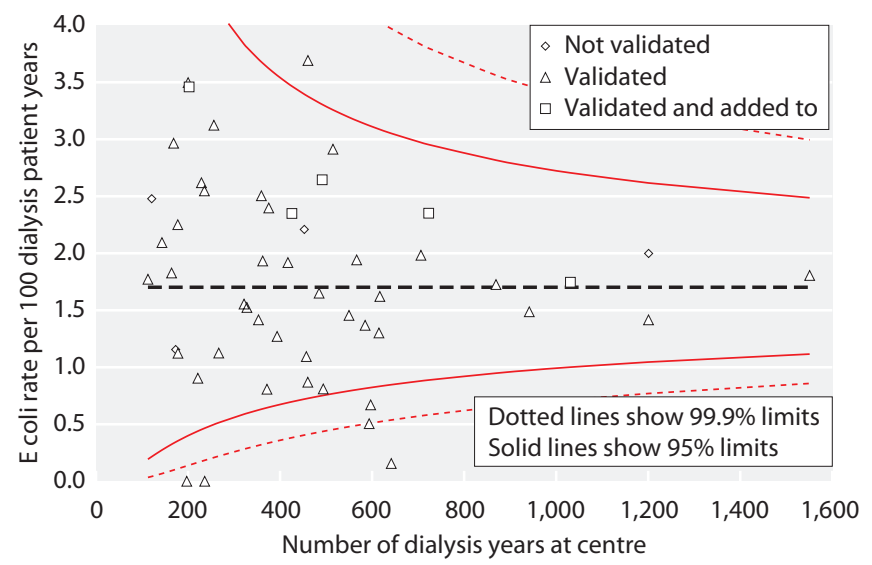

Fig. 10.6. Funnel plot of the Escherichia coli bacteraemia rate per 100 dialysis patient years by renal centre, 1st January 2015 to 31st December 2015

Dotted line depicts rate for whole cohort

plots each centre's estimated rate against the number of patient years to take into account the greater variation expected as centre size decreases. The rate in the HD population was twice as high compared to the PD population.

\section{Discussion}

This report presents data from one year of infections in patients receiving dialysis for ERF and continues the work of previous joint reports from PHE and the UKRR. It represents the second consecutive year where infections reported to PHE have been cross-checked with the UKRR database of patients receiving dialysis. For the first time, this allows a direct comparison with the previous year and removes the caution previously expressed due to differences in data collection and validation methods.

The rate of MRSA infections across England has remained stable year-on-year since 2011 following the earlier improvement in rates across England. This reflects the impact of increased awareness, training and screening. The enhanced attention given to this by dialysis units has resulted in this sustained improvement since reporting began in 2007/8 and represents a genuine success story.

This report presents the fourth full year of reporting of MSSA bacteraemia rates. The rate remains much higher than MRSA with a reporting rate 18 -fold higher. MSSA rates amongst dialysis patients has increased for the periods covered by each of the last three reports and while changes in reporting patterns undoubtedly account for some of this increase it does suggest that MSSA remains a significant issue in dialysis units in England. While no case-mix adjustment has been performed to take into account factors associated with catheter usage, combined data from this report and the 2015 vascular access report suggests that the presence of a central venous catheter remains a risk factor for development of staphylococcal infection. It also demonstrates that there is risk associated with an arteriovenous fistula. It is notable that there is much variation between centres in terms of MSSA infection rates. This may represent differences in screening programmes, access rates and methods of access care. Studying local variation in policies more closely may provide insight into the reasons for the variation in rates. Rates of MRSA have remained low whilst rates of MSSA have risen over the years. There is considerable incentive for hospital trusts to keep the rates of MRSA low, meaning much time, effort and resource is devoted to MRSA screening and eradication programmes.

This year's report includes further data on CDI and E.coli blood stream infection. CDI rates amongst dialysis patients remained stable. Antibiotic policies vary considerably between centres and there are no centre level data on antibiotic usage. Like MRSA there is considerable effort being made in all trusts to keep rates of CDI low with enhanced screening, isolation and change in antibiotic prescribing practices all being put in place. The national rate of E.coli bacteraemia amongst dialysis patients is similar to that observed last year. It is worth noting that Public Health England reported rises in 
incidence of MSSA and E. coli bacteraemia in the general population and so these increases are consistent with the overall trend in these infections [5].

Future reports will give more information regarding the long-term trends. The improved data accuracy and completeness that has resulted from linkage between PHE and UKRR databases will allow any trends to be more clearly identified. Antimicrobial resistance will also become an increasing focus. Public Health England launched a five year antimicrobial resistance strategy in 2014 [6]. Whilst there has been significant progress in reduction of the incidence of MRSA, the rise in multi drug resistant organisms represents a serious challenge to healthcare in the 21st century. For example, PHE is working with clinicians to develop guidance for screening and management of Carbapenemase-producing Enterobacteriaceae within dialysis facilities. NHS Improvement are leading on a strategy to halve gram negative blood stream infections by 2021 [7] with a focus on E. coli which accounts for about $55 \%$ of those. As part of that strategy, the UKRR will need to consider broadening data collection to other organisms and antibiotic usage as well as supporting improvement initiatives within renal services.

Conflicts of interest: the authors declare no conflicts of interest

\section{Acknowledgement}

The authors wish to acknowledge the assistance of renal centre colleagues for their assistance in providing the data to compile this report.

\section{References}

1 Steenkamp R, Rao A, Fraser S. UK Renal Registry 18th Annual Report (December 2015) Chapter 5: Survival and Causes of Death in UK Adult Patients on Renal Replacement Therapy in 2014: National and Centre-specific Analyses. Nephron. 2016;132 Suppl:111-44.

2 Bray BD, Boyd J, Daly C, Donaldson K, Doyle A, Fox JG, et al. Vascular access type and risk of mortality in a national prospective cohort of haemodialysis patients. QJM. 2012 Nov;105(11):1097-103.

3 Fluck R, Wilson J, Tomson CRV. UK Renal Registry 12th Annual Report (December 2009): chapter 12: Epidemiology of methicillin resistant Staphylococcus aureus bacteraemia amongst patients receiving dialysis for established renal failure in England in 2008: a joint report from the UK Renal Registry and the Health Protection Agency. Nephron Clin Pract. 2010;115 Suppl:c261-70.
4 Evans R, Caskey F, Fluck R, Crowley L, Davies J, Nsonwu O, et al. UK Renal Registry 18th Annual Report: Chapter 12 Epidemiology of Reported Infections amongst Patients Receiving Dialysis for Established Renal Failure in England 2013 to 2014: a Joint Report from Public Health England and the UK Renal Registry. Nephron. 2016;132 Suppl: 279-88.

5 https://www.gov.uk/government/uploads/system/uploads/attachment data/file/625784/Annual_epidemiological_commentary_2017.pdf

6 https://www.gov.uk/government/collections/antimicrobial-resistanceamr-information-and-resources

7 https://improvement.nhs.uk/resources/preventing-gram-negativebloodstream-infections/ 
